\title{
Noise Properties of the Boomerang Instrument
}

\author{
F. Piacentini ${ }^{a}$, P.A.R. Ade ${ }^{b}$, J.J. Bock $^{c}$, J.R. Bond $^{d}$, J. Borrill $^{e}$, A. Boscaleri ${ }^{f}$, \\ K. Coble ${ }^{g}$, C.R. Contaldi ${ }^{d}$, B.P. Crill ${ }^{h}$, P. de Bernardis ${ }^{a}$, G. De Troia ${ }^{a}$, P. Farese ${ }^{g}$, \\ K. Ganga ${ }^{i}$, M. Giacometti ${ }^{a}$, E. Hivon ${ }^{i}$, V.V. Hristov ${ }^{h}$, A. Iacoangeli ${ }^{a}$, A.H. Jaffe ${ }^{l}$, \\ W.C. Jones ${ }^{h}$, A.E. Lange ${ }^{h}$, L. Martinis ${ }^{m}$, S. Masi ${ }^{a}$, P. Mason ${ }^{h}$, P.D. Mauskopf ${ }^{n}$, \\ A. Melchiorrio ${ }^{o}$, T. Montroy ${ }^{g}$, C.B. Netterfield ${ }^{p}$, E. Pascale $^{f}$, D. Pogosyan ${ }^{d}$,

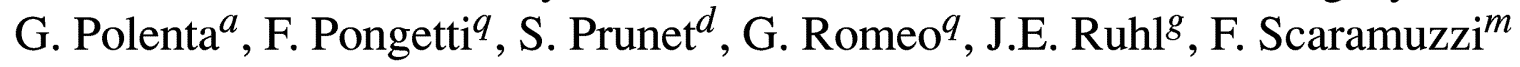 \\ ${ }^{a}$ Dipartimento di Fisica, Universitá La Sapienza, Roma, P.le A. Moro, 2, 00185, Italy. ${ }^{b}$ Queen Mary and \\ Westfield College, London, UK. ${ }^{c}$ Jet Propulsion Laboratory, Pasadena, CA, USA. ${ }^{d}$ C.I.T.A., University of \\ Toronto, Canada. ${ }^{e}$ N.E.R.S.C., LBNL, Berkeley, CA, USA. ${ }^{f}$ IROE-CNR, Firenze, Italy. ${ }^{g}$ Dept. of Physics, Univ. \\ of California, Santa Barbara, CA, USA. ${ }^{h}$ California Institute of Technology, Pasadena, CA, USA. ${ }^{i}$ IPAC, \\ Caltech, Pasadena, CA, USA. ${ }^{l}$ Department of Astronomy, Space Sciences Lab and Center for Particle \\ Astrophysics, University of CA, Berkeley, CA 94720 USA. ${ }^{m}$ ENEA, Frascati, Italy. ${ }^{n}$ Dept. of Physics and \\ Astronomy, Cardiff University, Cardiff CF24 3YB, Wales, UK. ${ }^{\circ}$ Nuclear and Astrophysics Laboratory, \\ University of Oxford, Keble Road, Oxford, OX $3 R H, U K .{ }^{p}$ Depts. of Physics and Astronomy, University of \\ Toronto, Canada. ${ }^{q}$ Istituto Nazionale di Geofisica, Roma, Italy.
}

\begin{abstract}
In this paper we report a short description of the BOOMERANG experiment explaining his scientific goal and the technologies implied. We concentrate then on the analysis of the noise properties discussing in particular the scan synchronous noise. Finally we present the calibration technique and the sensitivity of all the channels.
\end{abstract}

\section{INTRODUCTION}

BOOMERANG is a telescope devoted to the detection of structures in the Cosmic Microwave Background (CMB) radiation. The measure of structures in the primordial plasma (anisotropies in the CMB radiation, see [1] for a complete review) was the main goal of the recent observations in cosmology because gives information on the initial conditions that gave rise to the observed Universe. Moreover gives us the capability to tune the cosmological theories measuring the cosmological parameters [2], strongly correlated to the angular power spectrum of the CMB anisotropies (the statistical distribution of the size and the amplitude of the features in the CMB).

The CMB is observed now as Black Body emission at $T=2.726 \pm 0.005$ [3] which peaks in the microwave frequencies (80 to $250 \mathrm{GHz}$ ). The level of the expected fluctuation is of the order of $\Delta T / T<10^{-5}$, so a good experiment has to be able to detect temperature fluctuations of the order of $30 \mu \mathrm{K}$.

The angular dimensions of the most interesting features goes from a few degrees to a few arcminutes. Fluctuations in this angular range are expected to produce the maximum signal.

\section{THE INSTRUMENT}

Given those requirements BOOMERANG and other recent experiments have been developed with great care to make them reliable and to control systematics effects. BoomERANG $[4,5]$ succeeded in providing maps of the CMB radiation and the corresponding angular power spectrum with a high signal to noise ratio $[6,7]$.

The telescope uses 16 bolometric detectors made by a micromesh absorber with an indium bump-bonded NTD

CP616, Experimental Cosmology at Millimetre Wavelengths, 2 K1BC Workshop, edited by M. De Petris and M. Gervasi (C) 2002 American Institute of Physics 0-7354-0062-8/02/\$19.00 


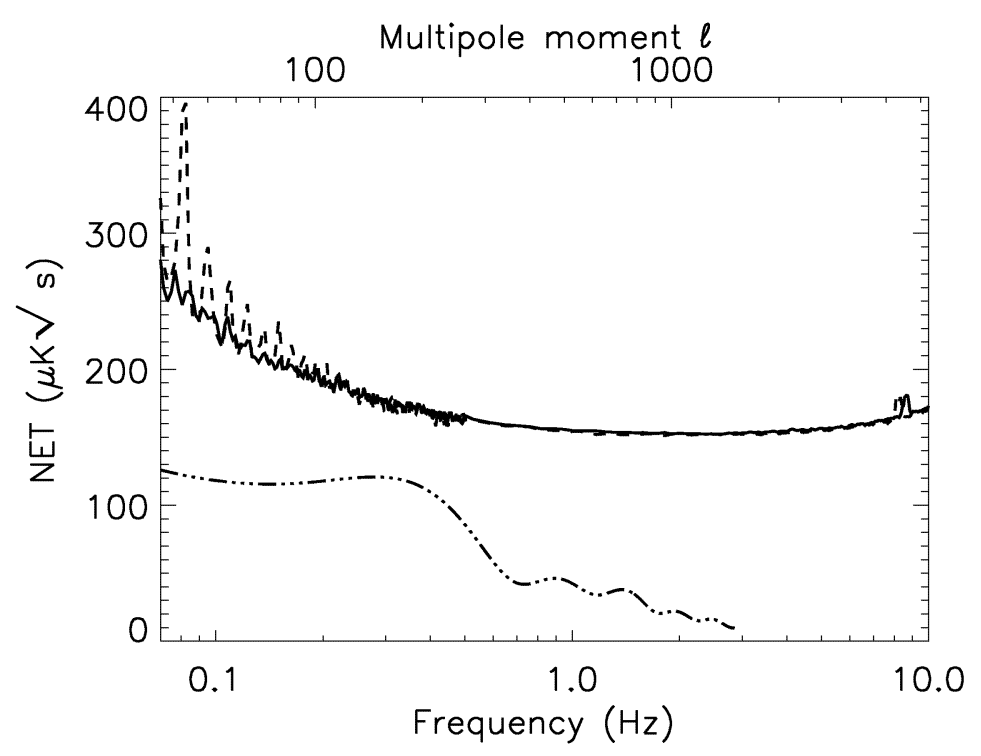

FIGURE 1. Typical power spectrum of the data and of the expected anisotropies for a BOOMERANG channel. The power spectral density of the data is plotted in thermodynamic temperature fluctuations of a $2.73 \mathrm{~K}$ blackbody.

Germanium thermistor [8] cooled at $280 \mu \mathrm{K}$ by a long duration cryogenic system $[9,10]$. The microwave frequencies are selected by band pass filters peaked at 90, 150, 240, $410 \mathrm{GHz}$ (with 20\% FWHM bandwidth) to map the CMB and to monitor spurious signals from the optics, the residual atmosphere and the galactic foregrounds.

Through the telescope each detector observes the sky in a proper direction. The full payload scans in azimuth with a smoothed triangular waveform ( 60 degrees peak to peak) so that each detector scans the sky. This movement, combined with the natural sky rotation during the day, determines the sky coverage.

During the scans structures in the sky are converted in temporal frequencies in the signal: anisotropies with a given multipole number are converted in signals with a corresponding electrical frequency in the $1-10 \mathrm{~Hz}$ range. The scan speed and the elevation angle, together with the noise and the frequency response of the detectors determine the angular range of sensitivity and the corresponding range in multipole numbers.

The elevation angle ranged during the flight between $40^{\circ}$ and $50^{\circ}$. The scan speeds we used ware of 1 and $2^{\circ} \mathrm{s}^{-1}$. The choice different scan modes and elevations was one of the several ways to allow control of systematic effects.

\section{NOISE PROPERTIES}

In figure 1 we report the power spectrum of the noise of a BOOMERANG channel (150A) for $1{ }^{\circ} \mathrm{s}^{-1}$ (continuum line) and $2{ }^{\circ} \mathrm{s}^{-1}$ (dashed line). In the same figure the expected angular power spectrum for a typical cosmological model is reported (dot-dashed line). The multipole moment scale on the top corresponds to the frequencies scale on the bottom in the case of $1^{\circ} \mathrm{s}^{-1}$ scan speed and $45^{\circ}$ of elevation. Comparing the noise power spectrum with the angular power spectrum makes evident how the noise characteristics of the detector are tuned to detect the expected CMB signal. The flat zone of minimum noise is between 0.4 and $3 \mathrm{~Hz}$ and the first three peaks in the angular power spectrum is of CMB anisotropies produce signal in the range $0.4-1.5 \mathrm{~Hz}$. The level of this white noise for all the channels is reported in table1 as Noise Equivalent Temperature (NET), together with other performances. The $1 / f$ noise knee is at $0.2 \mathrm{~Hz}$, corresponding to an angle in sky of 5 degrees. The rise in the Noise Equivalent Temperature at high frequencies is due to a decrease in sensitivity of the detectors where the transfer function starts to decrease. In fact high frequencies are cut off when the time scale starts to be comparable to the time constant of the detector. This rise in the noise corresponds to angles in the sky of the order of less than $0.1^{\circ}$, smaller than the size of the telescope beam.

The BoOMERANG readout electronics has a major role in the quality of the noise performance achieved. Each bolometer is AC biased with a differential smoothed square wave at $\sim 500 \mathrm{~Hz}$. The bias voltage is modulated by the high bolometer impedance to be measured. The signal goes trough a cold J-FETs pair to reduce its impedance and 
TABLE 1. Performances of the BoOMERANG channels: time constant, beam size and sensitivity. Only one channel (150A) was used for the first analysis [6] and four channels $(150 \mathrm{~A}, 150 \mathrm{~A} 1,150 \mathrm{~A} 2,150 \mathrm{~B} 2)$ were used in the latest analysis [7]. 150B was excluded due to the non stationary noise properties, 150B1 because didn't pass the jack-knife test for internal symmetry. The $90 \mathrm{GHz}$ and the $240 \mathrm{GHz}$ channels will soon be fully analyzed. The $410 \mathrm{GHz}$ channels doesn't contain cosmological information but have been used for dust contamination analysis [11]. The NET is computed at $1 \mathrm{~Hz}$. NET data of the $410 \mathrm{GHz}$ channels are missing because a reliable in-flight calibration for that channels is still in progress.

\begin{tabular}{lccc}
\hline Channel & $\begin{array}{c}\text { Time } \\
\text { constant (ms) }\end{array}$ & $\begin{array}{c}\text { Beam size } \\
\text { FWHM (arcmin) }\end{array}$ & $\begin{array}{c}\text { NET } \\
(\mu \mathbf{K} \sqrt{s})\end{array}$ \\
\hline 90A & 22.5 & $18 \pm 1$ & 145 \\
90B & 21.9 & $18 \pm 1$ & 137 \\
150A & 10.8 & $9.2 \pm 0.5$ & 130 \\
150B & 13.3 & $9.2 \pm 0.5$ & Variable \\
150A1 & 13.3 & $9.7 \pm 0.5$ & 231 \\
150A2 & 12.0 & $9.4 \pm 0.5$ & 158 \\
150B1 & 16.3 & $9.9 \pm 0.5$ & 196 \\
150B2 & 21.2 & $9.5 \pm 0.5$ & 184 \\
240A1 & 8.9 & $14 \pm 1$ & 221 \\
240A2 & 7.2 & $14 \pm 1$ & 166 \\
240B1 & 10.5 & $14 \pm 1$ & 250 \\
240B2 & 8.8 & $14 \pm 1$ & 792 \\
410A1 & 4.1 & $12 \pm 1$ & \\
410A2 & 9.9 & $12 \pm 1$ & \\
410B1 & 4.5 & $12 \pm 1$ & \\
410B2 & 4.3 & $12 \pm 1$ & \\
\hline
\end{tabular}

its sensitivity to inductive interferences, is amplified by a room temperature low noise differential pre-amplifier and band-pass filtered to remove out-of-band noise. A phase sensitive detector demodulates the signal synchronously with the bias, thus removing any offset and reducing the noise. Subsequent high-pass and low-pass filters get rid of residual $1 / f$ and high frequency noise.

The use of AC modulation and demodulation technique reduces $1 / f$ noise due to drifts in the bias amplitude, amplifier gain and heath sink temperature. The bias frequency has been selected in order to avoid beating with microphonic frequencies, thus reducing undesired lines in the noise power spectrum.

\section{Low frequency noise and calibration}

The noise power spectrum in the $2^{\circ} \mathrm{s}^{-1}$ scan mode has two peaks at the scan frequency and at the first harmonic, evidence for a strong component of Scan-Synchronous Noise (SNN). Those frequencies are well below the frequencies of interest for the sub-degree CMB anisotropy which is the target of this observation. Nevertheless is very important to have a reliable detection of the cosmic dipole signal (well known from COBE/DMR measurements [12]) for calibration. The cosmic dipole signal appears as a large scale linear gradient in the sky region observed and is converted into a signal at exactly the scan frequency by the telescope azimuthal movement.

The effect of the scan synchronous noise is clearly shown and compared to the expected dipole signal in time domain in figure 2. The source of this noise shouldn't be in the sky, because the sky signal doesn't change between fast and slow scan modes. It is probably a systematic effect in the instrument, more effective when the payload moves faster. The gyroscopes don't show significant different pendulations in the two scan modes, excluding the possibility of a signal in the sky excited by larger pendulations. Defining $\alpha$ as the angle in azimuth respect to the center of the scan and binning data from the evaporator thermometer for equal $\alpha$ bins, we detect a tiny coherent evaporator temperature variation in the $2^{\circ} \mathrm{s}^{-1}$ scan mode. The same effect is negligible in the $1^{\circ} \mathrm{s}^{-1}$ scan mode (see figure 3 ). Thermal effects are thus the first candidates as scan synchronous noise sources.

The calibration of the BoOMERANG telescope [13] is done using the signal from cosmic dipole: low-resolution $\left(1^{\circ} \times 1^{\circ}\right)$ maps are created for the two different scan modes separately; these maps are fit to a dipole model giving the 


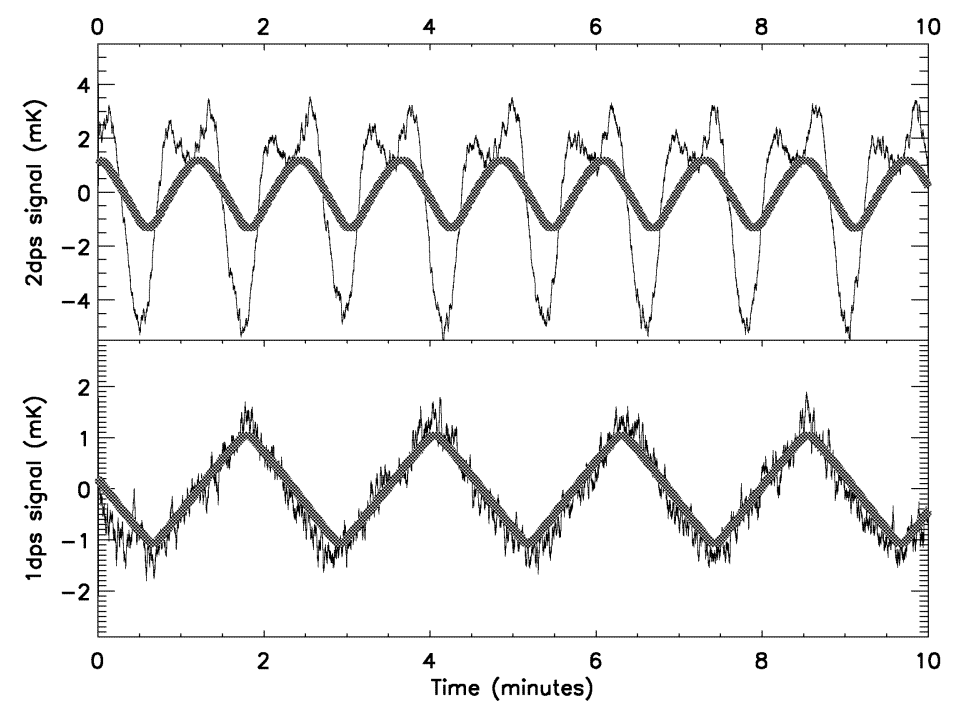

FIGURE 2. Comparison between data (thin line) and dipole simulations (thick light line) for the two different scan modes for the same channel. Data have been low pass filtered in order to display only the low frequency component. The dipole signal expected in each direction of observation has been computed from the COBE-DMR dipole parameters. In the $1^{\circ} \mathrm{s}^{-1}$ data (lower plot) data copy exactly the simulation, showing a very low component of scan synchronous noise. In the $2^{\circ} \mathrm{s}^{-1}$ data, the simulation differs clearly from signal. The discrepancy is due to a strong scan synchronous noise that appears only in the fast scan mode.
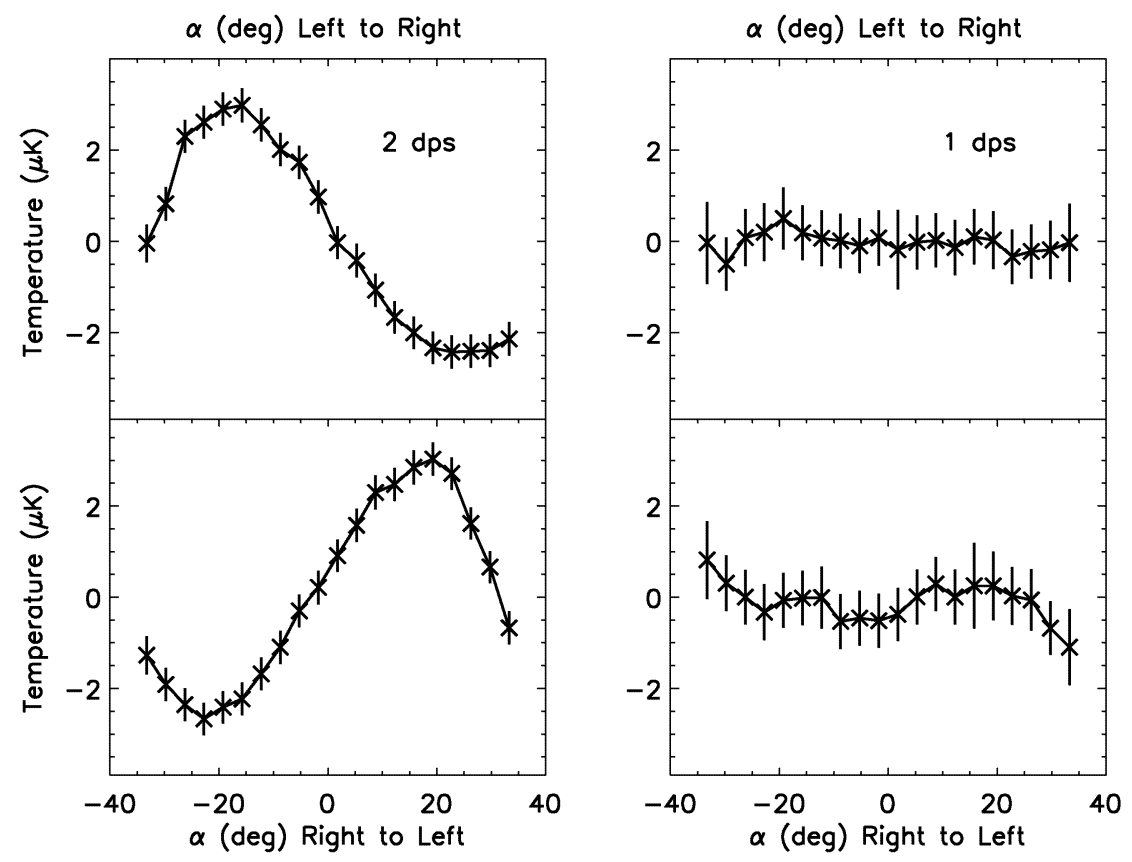

FIGURE 3. Temperature fluctuations of the evaporator binned for equal angles with respect to the center of the scan ( $\alpha$ ), for different scan modes. The top plots use data in the forward (left to right) part of the scans, the bottom plots in the reverse scans. Left plots are for $2^{\circ} \mathrm{s}^{-1}(2 \mathrm{dps})$ and right plots for $1^{\circ} \mathrm{s}^{-1}(1 \mathrm{dps})$ scan modes. The fluctuations are scan synchronous in the $2 \mathrm{dps}$ scan mode, while no coherence is present in the 1dps mode. Each plot results from integration of 24 hours of data. 
calibration constants for the channels sensitive to the CMB. Considering that the dipole signal is scan synchronous, any $1 / f$ noise and in particular scan synchronous spurious signal affects the dipole-based calibration. Anyway we know that in the slow scan mode the effect of scan synchronous noise is negligible (see figure 2). Even in the fast scan mode the receivers can be calibrated using the dipole signal, considering that the large scan synchronous noise is substantially reduced in the map-making process, because the scan synchronous signal is not exactly sky synchronous. In other words, making a map most of the scan synchronous noise is averaged out, reducing its effect. Moreover, observing that the scan synchronous noise at low frequencies $(90,150$ and $240 \mathrm{GHz})$ is well correlated to the scan synchronous noise in the $410 \mathrm{GHz}$ channels (where the CMB signal is negligible), a $410 \mathrm{GHz}$ map can be used as a template to remove the residual effect, thus producing a reliable dipole-based calibration for the low frequencies channels in the fast scan mode. The same procedure, applied to the $1^{\circ} \mathrm{s}^{-1}$ scan data, gives a correction of the order of $1 \%$. The two calibration constants, at 1 and $2^{\circ} \mathrm{s}^{-1}$ differ by $10 \%$ and this value is chosen as the calibration uncertainty, which is dominated by systematics. Consistency in the two calibrations provides a check for systematic effects, in particular shows that the results don't suffer for incorrect knowledge of the electronics transfer function at low frequency.

An other systematic effect, not directly related to $1 / f$ and scan synchronous noise, is the presence in the BOOMERANG maps of faint stripes at nearly constant declination. An equivalent effect is not reproduced in the simulated maps, varies in amplitude and phase between bolometers and is negligible in some of the channels. Several tests have been performed excluding the possibility of a signal in the sky. The effect has been taken into account in the map-making process, filtering it out in Fourier space. The same filter has been applied in any simulated map used in the Monte Carlo Spherical Harmonic Transform technique used to compute the angular power spectrum of anisotropies combining data from different channels [14].

\section{CONCLUSIONS}

The noise properties of the BOOMERANG instrument have been studied in detail before science release. Monitoring the full instrument and redundancy in the experimental setup (multi-frequency observations, different scan-speeds, long integration time) allowed control and understanding of spurious effects, thus giving great confidence in the BOOMERANG results $[6,7,15,16,17]$.

\section{ACKNOWLEDGMENTS}

BOOMERANG was supported by PNRA, Universitá di Roma La Sapienza and ASI in Italy, by NSF and NASA in USA and by PPARC in the UK. We thanks the $2 \mathrm{~K} 1 \mathrm{BC}$ conference organization for the very good job they did.

\section{REFERENCES}

1. White, M., Scott, D., and Silk, J., Annu. Rev. Astron. Astrophys., 32, 319-370 (1994).

2. Bond, J. R. et al., MNRAS, 291, L33 (1997).

3. Smoot, G. F. et al., Ap.J.L., 371, L1 (1991).

4. Piacentini, F. et al. (2002), accepted by Ap.J.Supp., astro-ph/0105148.

5. Crill, B. P. et al. (2002), in preparation.

6. deBernardis, P. et al., Nature, 404, 955-959 (2000), astro-ph/0004404.

7. Nettefield, C. B. et al. (2001), submitted to Ap.J.L., astro-ph/0104460.

8. Mauskopf, P. et al., Applied Optics, 36, 765 (1997).

9. Masi, S. et al., Cryogenics, 38, 319 (1998).

10. Masi, S. et al., Cryogenics, 39, 217-224 (1999).

11. Masi, S. et al., Ap.J.L., 553, L93-L96 (2001), astro-ph/0101539.

12. Kogut, A. et al., Ap.J., 419, 1-6 (1993).

13. Crill, B. P., Ph.D. thesis, Caltech, Pasadena, CA (2000).

14. Hivon, E. et al. (2001), submitted to Ap.J., astro-ph/0105302.

15. Lange, A. E. et al., Phys. Rev. D, 63, 4 (2001).

16. Jaffe, A. et al., Phys. Rev. Lett., 86, 3475-3479 (2001), astro-ph/0007333.

17. deBernardis, P. et al. (2001), accepted by Ap.J., astro-ph/0105296. 